\title{
TWEETHER project for W-band wireless networks
}

Claudio Paoloni ${ }^{1}$, François Magne ${ }^{2}$, Frédéric André ${ }^{3}$, Viktor Krozer ${ }^{4}$, Xavier Begaud $^{5}$, Sophie Kohler $^{3}$, Rosa Letizia $^{1}$, Quang Trung Le ${ }^{9}$, Marc Marilier ${ }^{6}$, Antonio Ramirez ${ }^{7}$, Ahmed Sabaawi ${ }^{1}$, Giacomo Ulisse ${ }^{4}$, Ruth Vilar ${ }^{8}$, Ralph Zimmerman $^{9}$

${ }^{1}$ Lancaster University, Lancaster LA1 4YW, UK email: c.paoloni@lancaster.ac.uk

${ }^{2}$ When-AB, Paris, France

${ }^{3}$ Thales Electron Devices, Velizy 78140, France

${ }^{4}$ Goethe University of Frankfurt, Frankfurt 60323, Germany,

${ }^{5}$ LTCI, CNRS, Télécom ParisTech, Université Paris-Saclay, France,

${ }^{6}$ OMMIC S.A.S., Limeil Brevannes 94453, France

${ }^{7}$ Fibernova Systems, Valencia 46022, Spain

${ }^{8}$ Universitat Politècnica de València, Valencia 46022, Spain,

${ }^{9}$ HF Systems Engineering GmbH, Kassel 34123, Germany

\begin{abstract}
The European Horizon 2020 project TWEETHER aims to make a breakthrough in wireless networks to overcome the congestion of the actual mobile networks and foster the new 5G networks. A European Consortium including four universities and five companies from four European countries is devoting a relevant effort to realize novel terminals and transmission hubs to operate in the W-band $(93-95 \mathrm{GHz})$. This paper will describe the advancement of the project.
\end{abstract}

Keywords-millimeter wave; wireless; traveling wave tube; backhaul; access

\section{INTRODUCTION}

The Horizon 2020 is a large research program of the EU to foster the progress by a collaborative approach among the best researchers in Europe. TWEETHER "Traveling wave tube based W-band wireless network with high data rate, distribution, spectrum and energy efficiency" [1] proposes a novel approach to exploit the millimeter wave portion of the spectrum for wireless communications. The challenge is to overcome the high atmospheric attenuation with an affordable front end. The technology at millimeter wave is not yet mature as it is at microwave frequencies. A relevant design and fabrication effort is on going to provide components suitable for outdoor wireless communications. In particular, the enabling device is a W-band high power traveling wave tube (TWT) that requires state of the art facilities and novel

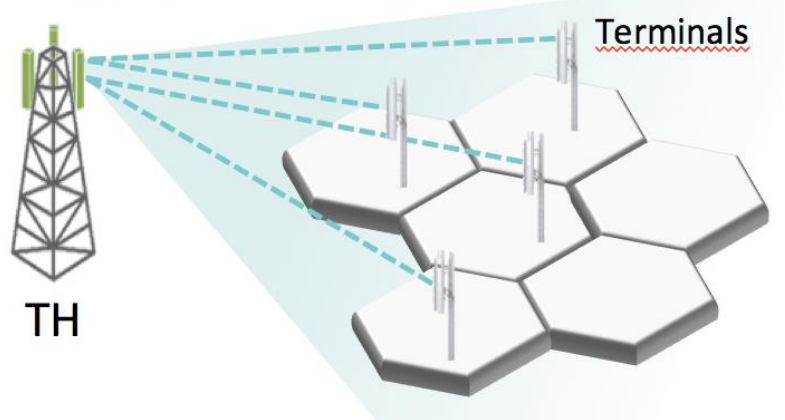

Fig. 1 Point to multipoint solutions.

\section{Millimeter WAVE FRONT END}

Most of the millimeter waves transmission systems are available for point to point (PtP) applications in V-band and E-band. Due to the limited output power available by solid state power amplifiers (SSPA), the use of high gain antennas is mandatory to achieve a useful range due to the strong atmospheric attenuation. The electronic at W-band is relatively new. GaN SSPAs are available with output power in the range of $1 \mathrm{~W}$. However, this power level is not sufficient for enabling the coverage of relatively wide area with internet wireless at millimeter wave frequencies. The novelty of TWEETHER project has been to introduce a traveling wave tube with saturate power of about $46 \mathrm{dBm}$ to use a low gain antenna to cover a defined sector from the transmission hub (TH). The omnidirectional coverage can then be obtained by 3 or 4 sectorial antennas. This approach will enable the first Point to Multipoint [2] (Fig.1) system at W band.

The paper will describe the last development of the project, including the chipset at W-band, the TWT performance and the system design to provide a new approach to wireless communication networks.

A purposely developed deployment model predicts a capacity density of $10 \mathrm{Gbps} / \mathrm{km}^{2}$ distributed over a circular sector with about $1 \mathrm{~km}$ radius. The excellent results satisfy the needs of both backhaul and access at high data rate.

\section{Acknowledgment}

The project has received funding from the European Union's Horizon 2020 research and innovation program under grant agreement no 644678 .

\section{References}

[1] TWEETHER website [Online]. Available: http://www.tweether.eu 
[2] R. Vilar, J. Martí and F. Magne, "Point to multipoint wireless backhaul systems for cost-effective small cell deployment," Microwave 